\title{
ARTICLE
}

\section{Vascular depression in older people with diabetes}

\author{
D. G. Bruce - G. Casey - W. A. Davis - S. E. Starkstein • \\ R. C. Clarnette • J. K. Foster • F. J. Ives • O. P. Almeida • \\ T. M. E. Davis
}

Received: 23 March 2006 / Accepted: 25 August 2006 / Published online: 13 October 2006

(C) Springer-Verlag 2006

\begin{abstract}
Aims/hypothesis Cerebrovascular disease may be causal or a vulnerability factor in late-onset depression and may explain the high rate of depression in older adults with diabetes. We explored a wide range of potential explanatory variables of depression in a longitudinal study of older diabetic subjects to investigate the vascular depression hypothesis in these patients.

Methods We recruited 207 subjects with diabetes selected for potential cognitive deficits from an existing observational cohort study (average age $75.7 \pm 4.6$ years, $52.2 \%$ men) for an assessment of depression using a standardised diagnostic instrument (Cambridge Examination for Mental Disorders of the Elderly - Revised). All subjects underwent a detailed
\end{abstract}

D. G. Bruce $(\bowtie) \cdot$ G. Casey $\cdot$ W. A. Davis $\cdot$ T. M. E. Davis School of Medicine and Pharmacology,

University of Western Australia, Fremantle Hospital,

P.O. Box 480, Fremantle, Western Australia 6959, Australia

e-mail: dbruce@cyllene.uwa.edu.au

S. E. Starkstein • O. P. Almeida

School of Psychiatry and Neurosciences,

University of Western Australia,

Fremantle, Western Australia, Australia

R. C. Clarnette

Department of Community and Geriatric Medicine,

Fremantle Hospital,

Fremantle, Western Australia, Australia

J. K. Foster

Ageing and Alzheimer's Unit, Edith Cowan University,

Perth, Western Australia, Australia

F. J. Ives

SKG Radiology, Hollywood Private Hospital,

Perth, Western Australia, Australia clinical assessment at baseline and at follow-up (after 7.5 \pm 1.1 years).

Results Major depression was present in 45 subjects $(21.7 \%)$ and minor depression in ten $(4.8 \%)$. A positive history of strokes and the presence of peripheral arterial disease were significantly associated with depression at the time of diagnosis. In a subsample of 93 cases who underwent structural neuroimaging, the presence of cerebral infarcts was also significantly associated with depression. Treatment with glucose-lowering therapy, higher serum cholesterol levels and difficulties with activities of daily living at baseline were significant predictors of depression at follow-up.

Conclusions/interpretation A history of cerebrovascular disease was strongly associated with depression and cerebrovascular risk factors were significant predictors of depression in older diabetic patients. Our findings are consistent with the hypothesis that the excess risk of depression in older diabetic patients is related to underlying cerebrovascular disease.

Keywords Cerebrovascular disease - Depression · Diabetic complications · Diabetes · Longitudinal study · Old age
Abbreviations
ABI
ankle : brachial index
ACR
albumin : creatinine ratio
ADL activities of daily living
CAMCOG Cambridge Cognitive test
CAMDEX Cambridge Examination for Mental Disorders of the Elderly - Revised
CVD cerebrovascular disease
DSM-IV Diagnostic and Statistical Manual of Mental Disorders, 4th edition 


\begin{tabular}{|c|c|}
\hline FDS & Fremantle Diabetes Study \\
\hline GHS & General Health Status Questionnaire \\
\hline IQCODE & Informant Questionnaire for Cognitive \\
\hline & $\begin{array}{l}\text { Decline in the Elderly } \\
\text { modified Barthel index }\end{array}$ \\
\hline IMSE & $\begin{array}{l}\text { Mini Mental State Examination } \\
\text { peripheral arterial disease }\end{array}$ \\
\hline
\end{tabular}

\section{Introduction}

Many studies indicate that diabetic subjects are at greater risk of depression than non-diabetic individuals from the general population [1-3]. A meta-analysis of 42 studies concluded that the prevalence of comorbid depression in diabetic subjects was $11 \%$ when depression was assessed by diagnostic interview and $31 \%$ when assessed by selfreport questionnaire [3]. The combination of diabetes and depression in older subjects increased several-fold the likelihood of chronic complications [4], disability [4] and death $[4,5]$. Depression in diabetes occurs most commonly in middle or younger age [6] but there is evidence that the prevalence in older patients is higher than expected [7,8].

Potential causes of depression in chronic disease include psychosocial factors related to the burden of disease [9] and/or pathophysiological changes in neuroendocrine or immune function $[10,11]$. In addition, late-life cerebrovascular disease may predispose to or precipitate depression [12-14]. Diabetes and hypertension, which are potent risk factors for cerebrovascular disease [15], may also contribute to an increased risk of depression through vascular mechanisms. This so-called vascular depression has a distinctive clinical phenotype that includes onset in later life, characteristic phenomenological features, and greater disability and more severe cognitive impairment than depression without cerebrovascular disease [16].

Most studies of depression in diabetes have used symptom inventories that tend to overdiagnose the condition in comparison with diagnostic interviews [3] and none has considered specific depression syndromes in older patients. This latter observation is important because, although major depression may be most strongly associated with clinical outcomes [17], subsyndromal depressive disorders are common in old age [18] and may carry an adverse prognosis [19].

We studied major and minor depression in a sample of older diabetic subjects from a well-categorised, longitudinal, observational study in order to determine whether cardiovascular risk factors and cerebrovascular disease were risk factors for depression and whether depression in older subjects with diabetes had the clinical features of vascular depression.

\section{Subjects and methods}

Subjects

The present sample was drawn from surviving, older participants in the Fremantle Diabetes Study (FDS). The FDS was a longitudinal, observational study of diabetic patients from a postcode-defined community of 120097 people. Of 2258 diabetic patients identified between 1993 and 1996, 1426 (63\%) were recruited to the FDS and 1294 (91\%) had type 2 diabetes. Identification and recruitment methods, sample characteristics, including classification of diabetes and details of non-recruited patients, have been described elsewhere [20, 21].

Between 1 February 2001 and 31 December 2002, all 587 surviving (as of 1 February 2001) members of the original FDS cohort (831 subjects), who would have been aged 70 years or over by 31 December 2002, were invited to participate in a study of cognitive function. Of these, 302 subjects (51.4\%) agreed to participate in the first stage of a two-stage cognitive assessment procedure (a cognitive screen that is described below) and 207 subjects underwent the second stage, a detailed cognitive assessment that included an assessment of depression (see Fig. 1 for a flow chart illustrating recruitment to the study). Of the 285 nonrecruited eligible survivors, 66 (23.2\%) died before recruitment, 71 (24.9\%) were not contactable and 148 refused to participate (12 [4.2\%] were unavailable, 66 [23.2\%] gave health reasons [dementia, old age or being too unwell] and 69 [24.1\%] offered no reason for non-participation). The present study sample $(n=207)$ comprises all 173 subjects selected because of a potentially high risk of cognitive impairment based on screening criteria (described below)

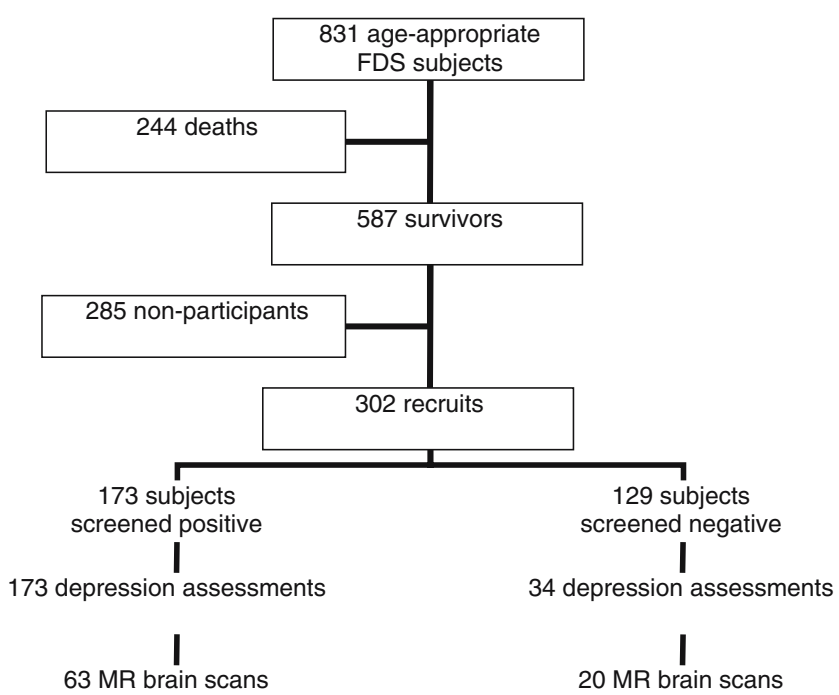

Fig. 1 Flow chart of subject recruitment 
plus a sample of subjects $(n=34)$ randomly selected from the remainder to provide information on subjects at low risk of cognitive deficits (Fig. 1). This sample represents an enriched sample for possible vascular depression, as cognitive deficits in diabetes are likely to have a cerebrovascular basis [22]. The study protocol was approved by the Human Rights Committee, Fremantle Hospital, and all subjects gave written informed consent.

\section{Clinical and laboratory methods}

Baseline assessment (1993-1996) At baseline a comprehensive history of diabetes and comorbid conditions was taken, a physical examination was performed and the patients provided fasting blood and urine samples for standard biochemical tests, including serum glucose, $\mathrm{HbA}_{1 \mathrm{c}}$, serum lipoproteins and albumin : creatinine ratio (ACR) [20-23]. Complications were identified using standard definitions. Peripheral sensory neuropathy was defined as a score of $>2 / 8$ on the clinical portion of the Michigan Neuropathy Screening Instrument [24]. A subject was defined as having retinopathy if any grade of retinopathy, including maculopathy, was detected by direct and/or indirect ophthalmoscopy in one or both eyes and/or more detailed assessment by an ophthalmologist. Nephropathy was defined as ACR $\geq 3.0 \mathrm{mg} / \mathrm{mmol}$ in an early morning urine sample [25]. Selfreported stroke and transient ischaemic attack were amalgamated with prior hospitalisations for these conditions to define baseline cerebrovascular disease (CVD) status. Patients were classified as having CHD if there was a self-reported history of, or hospitalisation for, myocardial infarction, angina, coronary artery bypass grafting, angioplasty and/or definite myocardial infarction on Minnesotacoded ECG [23]. The ankle : brachial index (ABI) was obtained from brachial and ankle systolic blood pressures using Doppler detection, and peripheral arterial disease (PAD) was defined as an ABI $\leq 0.90$ or the presence of a diabetes-related lower-extremity amputation [26]. Mobility impairment and activities of daily living (ADL) were assessed using the General Health Status questionnaire (GHS) [27], which includes questions on general mobility (selection of one of six states) and ADL (washing, dressing, eating and using the toilet) [28]. In this report, the data were dichotomised to normal mobility vs any impairment and normal ability with any ADL vs any impairment.

Follow-up assessment (2001-2002) At the follow-up assessment, the subjects underwent the same assessment of complications with the following differences: fundoscopy and ECGs were not performed and the GHS was not used. ADL was assessed using the modified Barthel index (MBI) supplemented by questions on the use of walking aids (dichotomised into no difficulty vs any difficulty with ADL and no use vs any use of a walking aid). The assessment was supplemented with a clinical examination carried out by experienced physicians (D.G.Bruce, R.C.Clarnette), which included a standardised neurological examination for evidence of CVD. This was refused by 26 subjects. In all cases hospital records were scrutinised for corroborative evidence, including results of previous brain imaging for CHD and CVD.

Cognitive screening procedure All subjects were assessed with the Mini Mental State Examination (MMSE) [29] and the Informant Questionnaire for Cognitive Decline in the Elderly (IQCODE) [30], and those with subjective memory complaints or who scored $<28 / 30$ on the MMSE or $>3.31$ on the IQCODE had a more comprehensive cognitive and mood assessment. Trained interpreters and appropriate versions of the MMSE (Italian, Croatian) were used when subjects were judged non-fluent in English.

Assessment of depression At baseline, depression was diagnosed based on the mood symptom inventory of the GHS. We had validated this diagnostic method previously in a comparable sample against standardised interviews using the Mini International Neuropsychiatric Interview and Diagnostic and Statistical Manual of Mental Disorders, 4th edition (DSM-IV) criteria for major and minor depression [5, 28] At follow-up, a research psychologist administered the revised version of the Cambridge Examination for Mental Disorders of the Elderly - Revised (CAMDEX) [31], a structured psychiatric interview for cognitive and mood disorders. The Italian language version of the CAMDEX was used in 34 non-fluent Italian speakers, administered by Italian-speaking psychologists [32]. The CAMDEX includes questions on depressed mood, loss of interest/pleasure, weight/appetite change, sleep disturbance, psychomotor retardation/agitation, fatigue, guilt, concentration and suicidal ideation and has high sensitivity, specificity and predictive values (over $90 \%$ ) for diagnosing major depressive disorder in a community sample of elderly people [33]. Based on responses to the appropriate CAMDEX items, diagnosis of major and minor depression were made using DSM-IV criteria. All the information and subsequent diagnoses were reviewed by a psychiatrist with experience of depression in clinical disorders (S.E. Starkstein). Medications, including antidepressants, were recorded and patients reported whether they had ever been treated with antidepressants.

Cognitive assessment Dementia was diagnosed based on DSM-IV criteria. The cognitive test battery included the Cambridge Cognitive test (CAMCOG), the CAMCOG Executive test and the MMSE score (derived from CAMCOG questions). The Brief Visual Memory Test Revised assessed visual learning and memory, and delayed 
recall scores are presented [34]. The Wechsler Memory Scale, Third Edition Word Lists [35] tested verbal memory, and delayed recall scores are presented. Subtests of the Wechsler Adult Intelligence Scale - Revised, Third Edition [36] assessed working memory (Letter-Number Sequence test) and spatial perception/problem-solving (Block Design). Verbal fluency was tested using the Controlled Oral Word Association test [37].

Radiological assessment Magnetic resonance (MR) brain scans were offered to 100 patients who screened positive and all 34 screen-negative patients who underwent detailed assessment (Fig. 1). Of these, 83 underwent standardised scans on a Siemens Vision $1.5 \mathrm{~T}$ scanner (Erlangen, Germany). A neuroradiologist (F.J. Ives) read them in blinded fashion for cerebral ischaemic infarcts, defined as focal hyperintensities on $\mathrm{T}_{2}$-weighted images $3 \mathrm{~mm}$ in size or larger and distinguished from dilated perivascular spaces by proton density scans. In a further 11 cases, previous brain computed tomography (CT) scan reports were available from the hospital records and these were classified by the presence or absence of brain infarction.

\section{Statistics}

The software package SPSS for Windows (version 11.5) was used for statistical analysis. Data are presented as proportion, mean $\pm \mathrm{SD}$, geometric mean (SD range) or, in the case of variables which did not conform to a normal or log-normal distribution, median (interquartile range).

For independent samples, multiple-sample comparisons of proportions were made with the $\chi^{2}$ test, for normally distributed variables ANOVA was used, and for nonnormally distributed variables the Kruskal-Wallis $H$-test was used. Two-sample comparisons of proportions were made using Fisher's exact test, for normally distributed variables Student's $t$ test was used, and for non-normally distributed variables the Mann-Whitney $U$ test was used. Multiple logistic regression analysis using forward conditional modelling ( $p<0.05$ for entry and $>0.10$ for removal) was performed to determine independent baseline associates of depression. All clinically plausible univariate variables with $p<0.20$ were considered for entry into the models.

\section{Results}

Sample characteristics and prevalence of depression

In comparison with the 302 recruits, the 285 eligible subjects who declined participation were significantly older $(77.4 \pm 6.3$ vs $75.0 \pm 4.6$ years; $p<0.001$ ), had a longer diabetes duration (11.9 [8.6-18.1] vs 10.3 [7.5-15.4] years; $p=0.001$ ), and were more likely to have died during the recruitment period ( 23.8 vs $4.3 \% ; p<0.001$ ), but the proportion of men in each group was similar ( 43.7 vs $48.3 \%$; $p=0.28$ ). There were no significant differences between the present study sample (207 subjects) and those who underwent the screening procedure only (in terms of age, gender, duration of diabetes, glycosylated haemoglobin $\left[\mathrm{HbA}_{1 \mathrm{c}}\right]$, living situation or ability with activities of daily living; all $p \geq 0.063$ ).

At the time of follow-up assessment, the study sample was aged $75.7 \pm 4.6$ years, $20.3 \%$ were aged 80 years and over, and $52.2 \%$ were male; only two patients lived in frail aged accommodation and a minority had difficulty with ADL (21.0\% had MBI scores below 95/100) or used a walking aid (22.1\%). Diabetes was of long duration (10.9 [8.3-15.5] years), 98.6\% had type $2 \mathrm{DM}, 49.2 \%$ had satisfactory $\mathrm{HbA}_{1 \mathrm{c}}$ levels $(\leq 7 \%)$ and $49.8 \%$ were obese $\left(\right.$ BMI $\left.>30 \mathrm{~kg} / \mathrm{m}^{2}\right)$. Major depression was present in 45 subjects $(21.7 \%)$ and ten $(4.8 \%)$ had minor depression. After excluding 16 subjects with dementia, 39/191 nondemented subjects had major depression $(20.4 \%)$ and ten had minor depression (5.2\%). Depression categories occurred with the same frequencies in women and men (women, 21.2\% major depression, 7.1\% minor depression; men, 22.2 and $2.8 \%$ respectively; $p=0.36$ ) and subjects aged 80 years or older had non-significantly higher prevalence rates of both depression subtypes (70-79 years, $20.6 \%$ major depression, $3.6 \%$ minor depression; $80+$ years, 26.2 and $9.5 \%$ respectively; $p=0.18$ ). Table 1 includes cognitive test results according to depression category. Subjects with major depression, but not those with minor depression, had significantly lower scores on cognitive tests, the strongest statistical effects being with subjective memory complaints, MMSE scores and delayed verbal and visual recall. Similar results were obtained when subjects with dementia were excluded (data not presented).

Cross-sectional associations with depression

Table 1 lists univariate clinical associations in the patient sample according to depression categories. There was no association with age, gender, marital status, duration of diabetes, glycaemic control, insulin therapy, BMI, blood pressure, history of hypertension, lipids, history of CHD, prior or current use of antidepressants, or current dementia. Minor depression was significantly associated with PAD and use of a walking aid. Major depression was associated with PAD, history and signs of stroke, recent physical exercise (negatively), low scores on the MBI and use of a walking aid. In multiple logistic regression analysis with depression (both major and minor) as the dependent 
Table 1 Cross-sectional demographic features, clinical associations and cognitive test results in 207 diabetic patients according to depression category

\begin{tabular}{|c|c|c|c|c|}
\hline & No depression & Minor depression & Major depression & $p$ value \\
\hline$n$ & 152 & 10 & 45 & \\
\hline Age at assessment & $75.1 \pm 4.4$ & $76.1 \pm 5.4$ & $76.5 \pm 4.6$ & 0.17 \\
\hline Sex $(\%$ male $)$ & 52.6 & 30.0 & 51.1 & 0.34 \\
\hline Duration diabetes (years) & $10.7(8.0-16.1)$ & $8.7(7.4-17.4)$ & $12.3(10.1-15.2)$ & 0.18 \\
\hline \multicolumn{5}{|l|}{ Diabetic treatment $(\%)$} \\
\hline Diet & 21.1 & 10.0 & 11.1 & 0.25 \\
\hline Oral agents & 55.9 & 50.0 & 62.2 & 0.68 \\
\hline Insulin ( \pm oral agents) & 23.0 & 40.0 & 29.7 & 0.37 \\
\hline BMI $\left(\mathrm{kg} / \mathrm{m}^{2}\right)$ & $28.4 \pm 4.3$ & $30.5 \pm 5.4$ & $27.9 \pm 3.8$ & 0.23 \\
\hline Systolic BP (mm Hg) & $151 \pm 22$ & $158 \pm 19$ & $156 \pm 28$ & 0.36 \\
\hline Diastolic BP (mm Hg) & $77 \pm 18$ & $74 \pm 12$ & $75 \pm 18$ & 0.80 \\
\hline History of hypertension (\%) & 63.9 & 70.0 & 79.1 & 0.17 \\
\hline $\mathrm{HbA}_{1 \mathrm{c}}(\%)$ & $7.3 \pm 1.3$ & $7.3 \pm 0.7$ & $7.4 \pm 1.4$ & 0.97 \\
\hline Fasting glucose $(\mathrm{mmol} / \mathrm{l})$ & $8.3 \pm 2.8$ & $7.8 \pm 1.8$ & $8.4 \pm 3.0$ & 0.83 \\
\hline Cholesterol (mmol/l) & $4.7 \pm 0.8$ & $5.3 \pm 1.5$ & $4.8 \pm 0.9$ & 0.19 \\
\hline HDL-cholesterol (mmol/l) & $1.3 \pm 0.4$ & $1.4 \pm 0.2$ & $1.3 \pm 0.5$ & 0.47 \\
\hline Serum triacylglycerol $(\mathrm{mmol} / \mathrm{l})$ & $1.5(0.9-2.4)$ & $1.7(1.0-2.9)$ & $1.6(0.9-2.8)$ & 0.55 \\
\hline Urinary albumin : creatinine ratio & $2.9(0.6-12.6)$ & $2.7(1.3-5.8)$ & $3.9(0.7-22.7)$ & 0.51 \\
\hline PAD $(\mathrm{ABI} \leq 0.90 ; \%)$ & 32.2 & $70.0^{\mathrm{a}}$ & $52.3^{\mathrm{a}}$ & $0.002^{\mathrm{e}}$ \\
\hline History of $\mathrm{CHD}(\%)$ & 32.8 & 50.0 & 40.0 & 0.43 \\
\hline History of CVD (\%) & 13.4 & 10.0 & $36.4^{\mathrm{b}}$ & $0.002^{\mathrm{e}}$ \\
\hline Confirmed CVD $(\%)^{\mathrm{c}}$ & 19.6 & 10.0 & $43.2^{\mathrm{b}}$ & $0.007^{\mathrm{e}}$ \\
\hline Physical CVD signs $(\%)^{\mathrm{c}}$ & 3.8 & 0 & $15.0^{\mathrm{a}}$ & $0.025^{\mathrm{e}}$ \\
\hline Neuropathy $(\%)$ & 27.2 & 60.0 & 27.3 & 0.08 \\
\hline Married (\%) & 65.3 & 70.0 & 61.4 & 0.83 \\
\hline Living alone $(\%)$ & 28.5 & 30.0 & 36.4 & 0.61 \\
\hline Exercise in last 3 months (\%) & 56.4 & 30.0 & $31.8^{\mathrm{b}}$ & $0.007^{\mathrm{e}}$ \\
\hline Barthel index & $100(98-100)$ & $97.5(93.5-100)$ & $97.5(88.3-100)^{\mathrm{b}}$ & $0.002^{\mathrm{e}}$ \\
\hline Use walking aid (\%) & 16.7 & $50.0^{\mathrm{b}}$ & $34.1^{\mathrm{a}}$ & $0.005^{\mathrm{e}}$ \\
\hline Antidepressant use (\%) & 11.4 & 20.0 & 13.3 & 0.70 \\
\hline Previous antidepressants (\%) & 16.7 & 30.2 & 30.0 & 0.11 \\
\hline Dementia $(\%)$ & 6.6 & 0 & 13.3 & 0.21 \\
\hline Subjective memory loss (\%) & 40.1 & 30.0 & $65.1^{\mathrm{b}}$ & $0.009^{\mathrm{e}}$ \\
\hline CAMCOG & $83.1 \pm 13.8$ & $88.4 \pm 4.4$ & $78.5 \pm 13.7^{\mathrm{a}}$ & 0.05 \\
\hline CAMCOG executive & $15.2 \pm 4.9$ & $16.3 \pm 3.8$ & $13.3 \pm 4.2^{\mathrm{a}}$ & $0.039^{\mathrm{e}}$ \\
\hline MMSE & $26(24-28)$ & $28(26-29)$ & $25(22-27)^{\mathrm{b}}$ & $0.011^{\mathrm{e}}$ \\
\hline Block design & $25.8 \pm 11.4$ & $25.7 \pm 11.0$ & $21.2 \pm 8.5^{\mathrm{a}}$ & 0.06 \\
\hline Delayed verbal recall $^{\mathrm{d}}$ & $4.1 \pm 2.6$ & $4.1 \pm 2.9$ & $2.7 \pm 1.9^{\mathrm{b}}$ & $0.013^{\mathrm{e}}$ \\
\hline Delayed visual recall & $5.4 \pm 3.0$ & $6.7 \pm 2.8$ & $4.1 \pm 2.6^{\mathrm{b}}$ & $0.012^{\mathrm{e}}$ \\
\hline Verbal fluency & $27.4 \pm 13.3$ & $30.4 \pm 15.1$ & $23.7 \pm 10.3$ & 0.18 \\
\hline Working memory & $7.2 \pm 2.9$ & $6.8 \pm 2.1$ & $6.1 \pm 2.5^{\mathrm{a}}$ & 0.07 \\
\hline
\end{tabular}

${ }^{\mathrm{a}} p<0.05$ compared with non-depressed group ${ }^{\mathrm{b}} p<0.01$ compared with non-depressed group

c181 subjects who underwent clinical examination (40 had major and 10 had minor depression)

${ }^{\mathrm{d}}$ Excluded 40 non-English-speaking subjects

e $p$ value denotes significant difference among the three groups

variable and considering all plausible variables with $p<0.20$ for entry into the model (age, stroke history, history of hypertension, PAD, cholesterol levels, duration of diabetes, prior antidepressant use, low MBI scores, exercise), significant independent variables associated with depression were PAD (odds ratio 2.27, 95\% CI 1.14-4.51; $p=$
$0.020)$ and physical exercise (protective effect) (odds ratio $0.35,95 \%$ CI $0.17-0.71 ; p=0.004)$. Removing functional factors (walking aid, physical exercise, low MBI scores) from the model to explore disease associations alone yielded both stroke history (odds ratio $2.39,95 \%$ CI $1.09-5.23 ; p=0.030$ ) and PAD (odds ratio $2.21,95 \% \mathrm{CI}$ 
$1.11-4.39 ; p=0.023)$ as independent variables associated with depression. We carried out the same analysis after excluding the subjects with dementia to test whether the association held in subjects without severe cognitive impairment and achieved very similar results, i.e. disease associations with depression were stroke history (odds ratio 2.54, 95\% CI 1.12-5.79; $p=0.026$ ) and PAD (odds ratio $2.27,95 \%$ CI $1.11-4.60 ; p=0.24$ ).

In the subset of patients with neuroimaging data $(82$ MRI scans, $11 \mathrm{CT}$ scans), nine of 68 non-depressed subjects (13.2\%), ten of 23 subjects with major depression $(43.5 \%)$ and one of two with minor depression $(50.0 \%)$ had neuroimaging evidence of cerebral infarcts $(p=0.003$ for major depression, $p=0.001$ for all depression). The results were similar when only MR scans were considered (data not shown).

\section{Longitudinal predictors of depression}

The relationship between baseline variables and subsequent depression (major and minor depression combined) are presented in Table 2. Significant univariate baseline predictors of subsequent depression were treatment with oral hypoglycaemic agents, higher serum total cholesterol levels, mobility impairment and ADL disability, whereas diet therapy for diabetes was protective (negative predictor). Of note, differences in baseline PAD prevalence approached statistical significance $(p=0.06)$. In multiple logistic regression and considering all plausible variables with $p<0.20$ for entry into the model (age, diet treatment, treatment with oral agents, systolic blood pressure, cholesterol, PAD, CHD, depression, exercise, mobility impairment, ADL disability), independent longitudinal predictors of depression were diet therapy (odds ratio $0.41,95 \%$ CI $0.19-0.85 ; p=0.017$ ), cholesterol (increase of $1 \mathrm{mmol} / \mathrm{l}$; odds ratio $1.46,95 \%$ CI $1.04-2.05 ; p=0.029)$ and ADL disability (odds ratio $3.63,95 \%$ CI 1.07-12.29; $p=0.038$ ).

Cognition in depressed subjects with stroke

The influence of stroke on cognitive test results was explored in depressed subjects (Table 3). Depressed subjects with a history of stroke had significantly lower scores on delayed visual recall (visual memory) and verbal fluency.

\section{Discussion}

We studied a group of older survivors from a wellcategorised, community-based longitudinal cohort study of patients with known diabetes in order to determine whether cerebrovascular disease was a risk factor for depression in older diabetic subjects. The recruitment strategy may have provided an enriched group for both depression and cerebrovascular disease as the sample contained patients with dementia and probably included cases with questionable dementia. The subjects had long-duration diabetes and many had atherosclerotic and microvascular complications, although most remained largely independent and nondisabled. We found that major depression occurred in approximately $20 \%$ of cases and minor depression in another 5\%. Given that the estimated prevalences of major and minor depression in older age are 1-4 and 4-13\% respectively $[38,39]$, these estimates are consistent with studies showing high rates of depression in older diabetic subjects [7]. Both longitudinal and cross-sectional data were consistent with the hypothesis that, in a substantial proportion of cases, depression had a cerebrovascular basis. Thus, the high rate of depression in older diabetic subjects may be partially explained by a high rate of vascular depression.

In the longitudinal study, independent baseline predictors of depression were the requirement for pharmacological glucose-lowering therapy, higher cholesterol levels and ADL disability. Each of these factors can be invoked to support a cerebrovascular causation of depression. Firstly, diabetes itself is a powerful independent predictor of stroke [40]. Most diabetic patients in the FDS experienced prolonged periods of hyperglycaemia because therapeutic intensification with glucose-lowering therapies was effected when $\mathrm{HbA}_{1 \mathrm{c}}$ was well above $7.0 \%$ [41]. This would be expected to substantially increase the risk of macrovascular or microvascular complications. In the present study, both longitudinal and cross-sectional analyses convincingly excluded CHD and microvascular disease from the causal pathway for depression, whereas CVD was implicated in the cross-sectional study. Second, there is increasingly strong evidence that elevated cholesterol levels are an independent risk factor for ischaemic stroke [42]. Finally, it is known that disability is a risk factor for depressive disorders [43] and we have demonstrated that both stroke and PAD are important predictors of ADL disability in diabetes [28].

Serum total cholesterol has been associated with depression and suicide risk, although the literature is difficult to interpret, low levels being associated with depression and suicide risk in some studies and high levels with suicide and non-response to antidepressant treatment in others [44]. It is noteworthy that in the present study baseline serum cholesterol levels were elevated, whereas at the time of depression assessment there was no difference, suggesting a dynamic relationship between dyslipidaemia and depression.

The cross-sectional study provided more direct evidence of a relationship between cerebrovascular disease and 
Table 2 Baseline data collected between 1993 and 1996 in 207 diabetic subjects diagnosed with depression in 2001-2002

\begin{tabular}{|c|c|c|c|}
\hline & No depression & Depression (minor or major) & $p$ value \\
\hline$n$ & $152(73.4 \%)$ & $55(26.6 \%)$ & \\
\hline Age & $67.6 \pm 5.2$ & $69.0 \pm 4.9$ & 0.09 \\
\hline Sex $(\%$ male $)$ & 52.6 & 49.1 & 0.75 \\
\hline Diabetes mellitus type $1(\%)$ & 2.6 & 0 & 0.58 \\
\hline Age at diagnosis (years) & $62.1 \pm 8.5$ & $63.4 \pm 8.0$ & 0.32 \\
\hline Duration of diabetes (years) & $3.0(0.5-8.8)$ & $5.0(1.6-8.0)$ & 0.35 \\
\hline \multicolumn{4}{|l|}{ Diabetic treatment $(\%)$} \\
\hline Diet & 41.4 & 23.6 & $0.022^{\mathrm{a}}$ \\
\hline Oral agents & 48.0 & 65.5 & $0.028^{\mathrm{a}}$ \\
\hline Insulin ( \pm oral agents) & 10.5 & 10.9 & 1.00 \\
\hline BMI $\left(\mathrm{kg} / \mathrm{m}^{2}\right)$ & $28.6 \pm 4.1$ & $29.1 \pm 3.9$ & 0.43 \\
\hline Waist circumference $(\mathrm{cm})$ & $97.6 \pm 11.0$ & $98.6 \pm 10.8$ & 0.59 \\
\hline Systolic BP (mm Hg) & $151 \pm 22$ & $157 \pm 21$ & 0.08 \\
\hline Diastolic BP (mm Hg) & $79 \pm 12$ & $81 \pm 10$ & 0.27 \\
\hline Taking BP-lowering medications (\%) & 53.9 & 54.5 & 1.00 \\
\hline $\mathrm{HbA}_{1 \mathrm{c}}(\%)$ & $7.3(6.3-8.8)$ & $7.8(6.6-8.6)$ & 0.36 \\
\hline FPG $(\mathrm{mmol} / \mathrm{l})$ & $8.3(6.8-10.6)$ & $8.7(7.0-10.9)$ & 0.49 \\
\hline Total cholesterol (mmol/l) & $5.4 \pm 1.0$ & $5.5 \pm 1.0$ & $0.02^{\mathrm{a}}$ \\
\hline HDL-cholesterol (mmol/l) & $1.07 \pm 0.32$ & $1.14 \pm 0.34$ & 0.59 \\
\hline Serum triacylglycerol $(\mathrm{mmol} / \mathrm{l})$ & $1.8(1.1-2.9)$ & $1.9(1.1-3.4)$ & 0.32 \\
\hline Taking lipid-lowering medications (\%) & 13.8 & 18.2 & 0.51 \\
\hline Taking regular aspirin $(\%)$ & 32.2 & 34.5 & 0.74 \\
\hline Urinary albumin : creatinine ratio & $2.1(0.7-6.4)$ & $2.5(0.7-8.6)$ & 0.25 \\
\hline $\mathrm{ACR} \geq 3.0 \mathrm{mg} / \mathrm{mmol}(\%)$ & 31.8 & 34.5 & 0.74 \\
\hline $\operatorname{PAD}(\mathrm{ABI} \leq 0.90 ; \%)$ & 19.9 & 32.7 & 0.06 \\
\hline CHD (\%) & 28.5 & 41.8 & 0.09 \\
\hline CVD (\%) & 9.2 & 14.5 & 0.31 \\
\hline Retinopathy (\%) & 12.2 & 18.5 & 0.26 \\
\hline Neuropathy $(\%)$ & 25.7 & 26.9 & 0.86 \\
\hline Married/de facto relationship (\%) & 73.7 & 70.9 & 0.73 \\
\hline Education ( $\%$ more than primary) & 78.1 & 70.9 & 0.36 \\
\hline English (\% not fluent) & 15.1 & 10.9 & 0.51 \\
\hline \multicolumn{4}{|l|}{ Ethnic background (\%) } \\
\hline Anglo-Celt & 75.0 & 72.7 & 0.72 \\
\hline Southern European & 19.1 & 6.4 & 0.84 \\
\hline Any exercise in previous 2 weeks (\%) & 84.2 & 74.5 & 0.15 \\
\hline \multicolumn{4}{|l|}{ Smoking status $(\%)$} \\
\hline Never & 50.7 & 42.6 & 0.34 \\
\hline Ex-smoker & 40.1 & 44.4 & 0.63 \\
\hline Current & 9.2 & 13.0 & 0.44 \\
\hline Alcohol (standard drinks/day) & $0(0-0.8)$ & $0.1(0-1.4)$ & 0.26 \\
\hline Depression $(\%)$ & 18.4 & 29.1 & 0.12 \\
\hline Mobility impairment (\%) & 11.3 & 24.1 & $0.041^{\mathrm{a}}$ \\
\hline ADL difficulty $(\%)$ & 3.3 & 12.7 & $0.017^{\mathrm{a}}$ \\
\hline
\end{tabular}

${ }^{\mathrm{a}} p$ value denotes significant difference

depression. Both PAD and stroke history were the strongest independent variables associated with depression and there were univariate associations with ischaemic infarcts in a subsample of patients with available neuroimaging. A link between PAD and depression in a cross-sectional clinicbased study was thought to be due to associated disability [45]. The Atherosclerosis Risk in Communities Study reported a modest association between baseline depressive symptoms and incident PAD [46]. However, CVD and PAD have a common underlying arterial pathology and shared risk factors and epidemiology [47], and PAD predicts future stroke as well as events in other vascular territories [48]. Thus, whilst the direction of our cross-sectional association between PAD and depression is unknown, the presence of PAD could denote cerebrovascular risk in these patients. We have previously demonstrated in FDS patients that age 
Table 3 Cognitive test scores in depressed subjects by history of previous stroke

\begin{tabular}{llll}
\hline & \multicolumn{2}{l}{ Depression (minor or major) } & \multirow{2}{*}{$p$ value } \\
\cline { 2 - 3 } & Stroke-free & Stroke history & \\
\hline CAMCOG & $82.5 \pm 10.8$ & $77.1 \pm 15.9$ & 0.16 \\
CAMCOG executive & $14.4 \pm 4.2$ & $12.8 \pm 4.4$ & 0.21 \\
MMSE & $26.5(22.3-28.0)$ & $24.5(23.3-26.8)$ & 0.18 \\
Block design & $23.3 \pm 9.0$ & $19.7 \pm 8.8$ & 0.20 \\
Delayed verbal recall & $3.5 \pm 2.5$ & $2.1 \pm 1.0$ & 0.09 \\
Delayed visual recall & $5.1 \pm 2.9$ & $3.3 \pm 2.1$ & 0.040 \\
Verbal fluency & $27.4 \pm 11.1$ & $19.2 \pm 10.6$ & 0.019 \\
Working memory & $6.4 \pm 2.6$ & $5.8 \pm 2.2$ & 0.48 \\
\hline
\end{tabular}

${ }^{a}$ Excluded non-English speaking subjects

and cholesterol predict incident PAD [26], providing an additional potential link between our longitudinal and cross-sectional findings.

In this sample, 16 of 44 subjects with depression at baseline were depressed at follow-up. In addition, few $(30.9 \%)$ of the depressed patients at follow-up reported ever receiving antidepressants. These data indicate that only a minority had recurrent or persistent depression and suggest that, for most, depression occurred for the first time in older age. Although we have no direct confirmatory data, this is consistent with the vascular depression hypothesis, whereby brain injury acquired through cerebrovascular disease leads to increased vulnerability in later life. In addition, depressed patients with a history of stroke had greater impairments in memory and verbal fluency than those without a history of stroke, consistent with findings in other patient groups classified as having vascular depression $[12,13]$.

This study has several important strengths and weaknesses. The study is unique in that the sample was recruited from an elderly, well-categorised, community-based sample of diabetic individuals with long-duration follow-up and that detailed data on a wide range of relevant potential explanatory variables were available. We also had detailed assessments of depression categories and cognitive function. The major weaknesses include the relatively low recruitment rate of available FDS survivors and a likely survivor effect. However, non-recruited survivors were older, many gave poor health as a reason for nonparticipation, and they had a higher mortality rate. Thus, they were likely to have more complications from diabetes, including cerebrovascular disease and depression, and the likely effect would be to weaken potential associations between depression and cerebrovascular disease. A second weakness relates to the recruitment strategy, which was designed to select subjects with cognitive impairment plus a sample of subjects with normal cognition. This probably led to an overestimate of the rate of depression and biased the study towards finding subjects with cerebrovascular disease. Finally, we do not have adequate data relating to depressive illnesses prior to FDS recruitment and the study is likely to have included subjects with both late-onset and recurrent depression. Again, this would be expected to weaken any associations that we found between vascular factors and depression.

The present study provides evidence that depression in older patients with diabetes is related to both vascular risk factors and cerebrovascular disease, and suggests that vascular causes explain the high rate of depression in older diabetic subjects. There are a number of clinical implications. Vascular depression is likely to be preventable by intensive cardiovascular risk factor management, but we have demonstrated that treatment of hypertension, dyslipidaemia and hyperglycaemia in our patients is frequently suboptimal [23, 41]. Major depression in late life is often recurrent and disabling and requires active management, yet is often undiagnosed and undertreated [43]. In the present study fewer than $15 \%$ of patients with major depression were taking antidepressant drugs, indicating inadequate treatment of depression.

An integrated, collaborative approach among health-care workers from primary care and diabetes and mental health services has been demonstrated to be more effective in treating older diabetic patients with depression [49]. Future research should consider whether depressive illnesses can be prevented by intensive management of cardiovascular risk factors in these patients. Combining effective cardiovascular risk-factor reduction with depression treatment could lead to a reduction in the recurrence rate of depression and could also reduce the excess risk of disability and death associated with depression $[4,5]$.

Acknowledgements This study was funded by grants from the National Health and Medical Research Committee of Australia and Diabetes Australia Research Trust.

Duality of interest The authors are unaware of any conflicts of interest.

\section{References}

1. Gavard J, Lustman P, Clouse RER (1993) Prevalence of depression in adults with diabetes. Diabetes Care 16:1167-1178

2. Eaton WE (2002) Epidemiologic evidence on the comorbidity of depression and diabetes. J Psychosom Res 53:903-906

3. Anderson RJ, Freedland KE, Clouse RE, Lustman PJ (2001) The prevalence of comorbid depression in adults with diabetes: a meta-analysis. Diabetes Care 24:1069-1078

4. Black SA, Markides KS, Ray LA (2003) Depression predicts increased incidence of adverse health outcomes in older Mexican Americans with type 2 diabetes. Diabetes Care 26:2822-2828 
5. Bruce DG, Davis WA, Starkstein SE, Davis TME (2005) A prospective study of depression and mortality in patients with type 2 diabetes: the Fremantle Diabetes Study. Diabetologia 48:2532-2539

6. Egede LE, Zheng D (2003) Independent factors associated with major depressive disorder in a national sample of individuals with diabetes. Diabetes Care 26:104-111

7. Bell RA, Smith SL, Arcury TA, Snivelly BM, Stafford J, Quande SA (2005) Prevalence and correlates of depressive symptoms among rural older African Americans, native Americans and whites with diabetes. Diabetes Care 28:823-829

8. Bruce DG, Casey GP, Grange V et al (2003) Cognitive impairment, physical disability and depressive symptoms in older diabetic patients: the Fremantle Cognition in Diabetes study. Diabetes Res Clin Pract 61:59-67

9. Lin EH, Katon W, Von Korff M et al (2004) Relationship of depression and diabetes self-care, medication adherence and preventive care. Diabetes Care 27:2154-2160

10. Checkley S (1996) The neuroendocrinology of depression and chronic stress. Br Med Bull 52:597-617

11. Herbert TB, Cohen S (1993) Depression and immunity: a metaanalytic review. Psychol Bull 113:472-474

12. Krishnan KRR, Hays JC, Blazer DG (1997) MRI-defined vascular depression. Am J Psychiatry 154:497-500

13. Alexopoulos GS, Meyers BS, Young RC, Campbell S, Sibersweig D, Charlson M (1997) The "vascular depression" hypothesis. Arch Gen Psych 54:15-22

14. Robinson RG (2003) Poststroke depression: prevalence, diagnosis, treatment and disease prevention. Biol Psychiatry 54:376-387

15. Loug S, Melamed E, Cahane E, Carmen A (1973) Hypertension and diabetes as risk factors in stroke patients. Stroke 4:751-759

16. Alexopoulos GS, Meyers BS, Young RC, Kakuma T, Silbersweig D, Charlson M (1997) Clinically defined vascular depression. Am J Psychiatry 154:562-565

17. De Groot M, Anderson R, Freedland KL, Clouse RE, Lustmnan PJ (2001) Association of depression and diabetes complications: a meta-analysis. Psychosom Med 63:619-630

18. Lavretsky H, Kumar A (2002) Clinically significant non-major depression. Old concepts, new insights. Am J Geriatr Psychiatry 10:239-255

19. Blazer DG (2003) Depression in late life: review and commentary. J Gerontol Med Sci 56A:249-265

20. Davis TM, Zimmet P, Davis WA, Bruce DG, Fida S, Mackay IR (2000) Autoantibodies to glutamic acid decarboxylase in diabetic patients from a multi-ethnic Australian community: the Fremantle Diabetes Study. Diabetic Med 17:667-674

21. Bruce DG, Davis WA, Davis TME (2000) Glycemic control in older diabetic patients in the Fremantle Diabetes Study. J Am Geriatr Soc 48:1449-1453

22. Cukierman T, Gerstein HC, Williamson JD (2005) Cognitive decline and dementia in diabetes - systematic overview of prospective observational studies. Diabetologia 48:2460-2469

23. Davis TME, Davis W, Mulder JW, Fortune P, Bruce DG (2004) Silent myocardial infarction and its prognosis in a communitybased cohort of diabetic patients: the Fremantle Diabetes Study. Diabetologia 47:395-399

24. Feldman EL, Stevens MJ, Thomas PK, Brown MB, Canal N, Greene DA (1994) A practical two-step quantitative clinical and electrophysiological assessment for the diagnosis and staging of diabetic neuropathy. Diabetes Care 17:1281-1289

25. Davis WA, Knuiman MW, Hendrie D, Davis TME (2005) Determinants of diabetes-attributable non-blood glucose-lowering medication costs for type 2 diabetes: the Fremantle Diabetes Study. Diabetes Care 28:329-336

26. Norman PE, Davis WA, Bruce DG, Davis TME (2006) Peripheral arterial disease and risk of cardiac death in type 2 diabetes: the Fremantle Diabetes Study. Diabetes Care 29:575-580
27. Gudex C, Kind P (1989) The QALY toolkit. Centre for Health Economics, University of York, York

28. Bruce DG, Davis WA, Davis TME (2005) Longitudinal predictors of reduced mobility and physical disability in patients with type 2 diabetes mellitus: the Fremantle Diabetes Study. Diabetes Care 28:2441-2447

29. Folstein M, Folstein S, McHugh PR (1975) Mini-mental State: a practical method for grading the cognitive state of patients for the clinician. J Psychiatr Res 12:189-198

30. Jorm AF (1994) A short form of the Informant Questionnaire on Cognitive Decline in the Elderly (IQCODE): development and cross-validation. Psychol Med 24:145-153

31. Roth M, Tym E, Mountjoy CQ et al (1986) CAMDEX. A standardised instrument for the diagnosis of mental disorder in the elderly with special reference to the early detection of dementia. Br J Psychiatry 149:698-709

32. Neri M, Andermarcher E, Spano A et al (1992) Validation study of the Italian version of the Cambridge Mental Disorders of the Elderly Examination: preliminary findings. Dementia 3:70-77

33. Girling DM, Barkley C, Paykel ES et al (1995) The prevalence of depression in a cohort of the very elderly. J Affect Dis 34:319-329

34. Benedict RHB (1997) Brief visual memory test - revised. Psychological Assessment Resources, Inc, Odessa, FL

35. Wechsler D (1997) Wechsler memory scale, Third edn. Psychological Corporation, New York

36. Wechsler D (1997) Wechsler adult intelligence scale, III edn. Psychological Corporation, San Antonio

37. Spreen O, Benton AL (1977) Manual of instructions. Neurosensory center comprehensive examination for aphasia. 1977 Revision. University of Victoria, Victoria, BC

38. Palsson S, Skoog I (1997) The epidemiology of affective disorders in the elderly: a review. Int Clin Psychopharmacol 12:S3-S13

39. Beekman ATF, Copeland JRM, Prince MJ (1999) Review of community prevalence of depression in late life. Br J Psychiatr 174:307-311

40. Kissela BM, Khouri J, Kleindorfer D et al (2005) Epidemiology of ischemic stroke in patients with diabetes: the Greater Cincinnati/ Northern Kentucky Stroke Study. Diabetes Care 28:355-359

41. Davis TME, Davis WA, Bruce DG (2006) Glycaemic levels triggering therapeutic intensification in type 2 diabetes in the community: the Fremantle Diabetes Study. Med J Aust 184:325-328

42. Amarenco P, Labreuche J, Lavalee P, Touboul P-J (2004) Statins in stroke prevention and carotid atherosclerosis: systematic review and up-to-date meta-analysis. Stroke 35:2902-2909

43. Alexopoulos GS (2005) Depression in the elderly. Lancet 365:1961-1970

44. Camus V, Kraehenbuhl H, Preisig M, Bula CJ, Waeber G (2004) Geriatric depression and vascular diseases: what are the links? J Affect Dis 81:1-16

45. Arseven A, Guralnik JM, Liu K, McDermott MM (2001) Peripheral arterial disease and depressed mood in older men and women. Vasc Med 6:229-234

46. Wattanakit K, Williams JE, Schreiner PJ, Hirsch AT, Folsom AR (2005) Association of anger proneness, depression and low social support with peripheral arterial disease: the Atherosclerosis Risk in Communities Study. Vasc Med 10:199-206

47. Rothwell PM, Coull AJ, Silver LE et al (2005) Population-based study of event-rate, incidence, case fatality, and mortality for all acute vascular events in all arterial territories (Oxford Vascular Study). Lancet 366:1773-1783

48. Doobay AV, Anand SS (2005) Sensitivity and specificity of the ankle-brachial index to predict future cardiovascular outcomes: a systematic review. Arterioscler Thromb Vasc Biol 25:1463-1469

49. Williams J, Katon W, Lim EHB et al (2004) Effectiveness of depression care management for older adults with coexisting depression and diabetes mellitus. Ann Int Med 140:1015-1024 